\title{
Assessment of Patient Comfort During Palliative Sedation: Is it always Reliable?
}

\author{
R. Deschepper, J. Bilsen, and S. Laureys
}

\section{Introduction}

"When death knocks at the door of our ward, we do not easily open the door", an intensivist once said. In the intensive care unit (ICU) and emergency department, care is strongly focused on cure and resuscitation. Notwithstanding the technological progress made in intensive and emergency medicine, a substantial number of the patients admitted to the ICU cannot be saved. In these cases, it is important to make a timely shift from curative efforts to palliative care, so that futile and burdensome interventions can be avoided. When death becomes imminent, a major concern of the family members and caregivers is to assure maximal comfort during the dying process. A central aspect of good end-of-life care is to keep the patient, as much as possible, free of pain and other kinds of distress. However, many critically ill patients often suffer from symptoms such as pain and delirium. More than $50 \%$ of critically ill patients in the ICU experience moderate to severe pain and pain in critically ill patients often remains untreated [1].

On some occasions, patients are unable to communicate or give signs of discomfort and in these circumstances ensuring comfort is very challenging. This is the case, for example, with patients who are in a coma and patients in a vegetative/unresponsive state. Communication is also limited with patients who have been diagnosed as minimally conscious, locked-in syndrome or late-stage dementia and with newborns. Patients may also be unable to communicate because of treatments, such as intubation and administration of neuromuscular blocking agents. In all these patients, assessment of comfort is challenging. Although it might sound obvious, it is important not to overlook the fact that the inability to communicate,

\footnotetext{
R. Deschepper · J. Bilsen

Department of Public Health, Vrije Universiteit Brussel, 1090 Brussel, Belgium

S. Laureys $\square$

Coma Science group, Cyclotron Research Centre and Neurology Department, University and University Hospital of Liège, Sart Tilman B35, 4000 Liège, Belgium

e-mail: steven.laureys@ulg.ac.be
} 
verbally or non-verbally, does not negate the possibility that an individual is experiencing pain or other kinds of distress and is in need of appropriate treatment to ensure optimal comfort.

\section{Box 1:}

\section{Core Elements in Guidelines on Palliative Sedation}

\section{- Indications for palliative sedation}

- Refractory symptoms leading to unbearable suffering such as intolerable pain, dyspnea and delirium [11, 12, 23, 35]

- Types of palliative sedation

- Degree: mild, intermediate and deep [15]

- Continuity: from intermittent to continuous [15]

- Ethical principles

- Palliative sedation is normal medical practice and must be clearly distinguished from the termination of life [35]

- Proportionality: the degree of sedation must not be deeper than necessary to relief suffering [11, 12, 23, 35]

- Palliative sedation will not (usually) hasten death (and that is certainly not the intention) $[12,15]$

- Administration of drugs

- Titration to the minimum level of consciousness reduction necessary to render symptoms tolerable [16, 23, 24]

- Lack of consensus

- "No good evidence exists to strongly recommend one medication over any other of those commonly used in continuous palliative sedation therapy" [16]

- "Midazolam is the drug of first choice" [24]

\section{- Monitoring of palliative sedated patients}

- Aspects requiring monitoring: [16]

- Relief of suffering

- Level of consciousness (depth of sedation)

- Adverse effects of sedation

- Guidelines' evaluations of the usefulness of monitoring scales

- "There are no scales available to assess the patient's comfort during continue sedation" [24]

- "Monitoring (observational) scales exist but the usefulness of these scales has not been proven" [16]

_ "Presently no particular scale can be recommended" [16, 24]

- "Scales involving administration of painful stimuli are not acceptable" [16]

- Frequency of monitoring: every 20 minutes until adequate sedation has been reached 
Patient comfort can often be achieved by 'conventional' pharmacological drugs such as opioids or other symptom-controlling drugs. However, in cases of severe pain caused by withdrawal of life-sustaining treatment, for example, or where refractory symptoms lead to unbearable suffering such as intolerable pain, dyspnea and delirium, a more drastic option may be chosen, known as palliative sedation (Box 1). In these cases, comfort is sought by reducing the patient's level of consciousness [2, 3]. Palliative sedation is ethically controversial because it is viewed by some as a kind of 'slow euthanasia' and because patients are not always involved in the decision-making. Another point of controversy is whether or not palliative sedation has a life-shorting effect and therefore should be considered to be an endof-life decision [4].

Some studies have pointed out that palliative sedation is an effective method that does not shorten survival time, but the topic creates controversy [2, 5]. However, the practice of palliative sedation has increased substantially and seems to have established its place in end-of-life care. The incidence of palliative sedation is not easily measured, partly because there are several definitions and alternative terms in use, such as 'terminal sedation' and 'continuous sedation until death', to describe this practice [6]. However, available studies indicate that the practice of palliative sedation is increasing in hospitals, nursing homes and the home care setting. The overall reported incidences vary between $7 \%$ and $17 \%$ of all deaths [7]. It is assumed that patients who are sedated according to the current standards of care and the guidelines for palliative sedation are unaware of their clinical situation and, therefore, do not experience symptoms of discomfort, such as dyspnea, delirium, and other distressing conditions that are common during the terminal phase. However, a critical evaluation based on more recent evidence raises the question of whether the current assessments of suffering and awareness are accurate enough. Our concerns are based on three types of problem. First, the assessment of comfort in dying patients is challenging; second, patients are sometimes mistakenly considered to be unaware, and, third, the titration of drugs is difficult.

\section{Problems with Assessment of Comfort in Dying Patients}

Pain is always subjective. Therefore, the gold standard for detecting distress is patient self-reporting. However, in the case of palliative sedation, patients are usually unable to communicate whether or not they are still in distress or still (partially) aware of what is happening around them. In these cases, caregivers have to rely on observable signs, such as facial expression. In newborns, for instance, tongue protrusion and licking the lips can be considered a positive affective expression, whereas brow wrinkling and opening the eyes wide are usually considered as facial expressions of negative affect [8]. To achieve a more reliable assessment, several instruments, such as the Visual Analog Scale for pain, are based on these observable signs. Some scales have been developed for non-communicative patients as well, including the Critical Care Pain Observational Tool (CCPOT) [9], the Behavioral Pain Scale (BPS) [10], and the Richmond Agitation-Sedation Scale (RASS) [11] 
but several problems have been reported. A well-documented problem is that these scales cannot detect pain and awareness in all patients, for example because they depend on inferences made from patients' motor responses [12, 13].

Another problem is that these scales have only been partially validated for dying patients, and in most cases not at all [14]. In the guidelines on palliative sedation it is acknowledged that the efficacy and safety of palliative sedation is not sufficiently understood and that the usefulness of these observational scales has not been proven $[15,16]$. These findings cause even more concern considering the evidence that family members of patients often have different perceptions of the patient's comfort and his/her quality of dying during palliative sedation than do caregivers. While family members tend to overestimate pain, caregivers often underestimate it [17]. Furthermore, assessment discrepancies between nurses and physicians often occur [18, 19]. Moreover, non-clinical factors, such as characteristics of both the patient and the caregiver, play a role. For example, paramedical professionals, religious correspondents and older caregivers more often believe that patients in vegetative or minimally conscious states may be experiencing pain [19]. In a study of patients coming to the emergency department with pain-related complaints, older men were less likely than younger men to receive an analgesic or an opioid regardless of pain severity. Older adults were generally less likely to receive pain treatment. However, older women with severe pain were more likely to receive treatment than younger women with severe pain. These results suggest an interaction between age, gender, and pain severity on pain treatment [20].

Physiological parameters such as respiratory frequency, heart rate, blood pressure, pupillary diameter and skin conductance, are also often used as indicators for discomfort but these are not always reliable either [21]. Furthermore, once a decision has been made to provide only palliative care, measuring these parameters is usually considered to be a futile and therefore unnecessary intervention, which is likely to be considered more of an obstacle to a quiet dying process than helpful for the patient.

When patients are considered completely unconscious, they are often considered unable to experience any kind of pain or emotion [22]. However, this topic is still controversial and poses clinical and ethical challenges [8]. In cases of painful interventions and especially after decisions to withdraw life-sustaining support, such as mechanical ventilation or artificial hydration and nutrition, these patients are, therefore, sometimes left without administration of opioids or other analgesic. Mistakenly considering a patient as unaware is a pitfall that may deprive a patient of optimal comfort medication. Therefore, the premise of unawareness is a crucial aspect in critically ill or dying patients. 


\section{Problems with Assessment of Awareness}

Given the importance of the status of unawareness in comfort care, a reliable assessment of awareness is pivotal. However, in recent years, doubts have arisen as to whether patients labeled 'unconscious' really are completely insensate and unaware. Studies in different types of patient and setting, which critically reviewed awareness, have consistently reported that persons were, in contrast to what was assumed by the caregivers, not always (completely) unaware. For example, several studies showed that patients diagnosed as being in a vegetative state (now also called 'unresponsive wakefulness syndrome') did show (minimal) clinical signs of conscious awareness in about $40 \%$ of cases [23]. In some cases, the purportedly unconscious patient could even reliably generate appropriate electroencephalographic (EEG) responses to two distinct commands [24] and occasionally was even able to establish basic communication with 'yes' or 'no' answers using functional magnetic resonance imaging (fMRI) [25]. These observations proved that some minority of clinically diagnosed unresponsive patients displayed some residual cognitive function and conscious awareness that even skilled caregivers were not able to recognize [26].

Furthermore, patients with locked-in syndrome may be mistakenly considered unconscious. In a survey of 44 patients with locked-in syndrome, the time elapsed between brain insult and the diagnosis of locked-in syndrome was on average 2.5 months (78 days). Several patients had not been diagnosed correctly for more than 4 years. It has been stated that this delay in the diagnosis of locked-in syndrome mainly reflected initial misdiagnosis [27].

In rare cases, patients also have reported awareness during general anesthesia. Light anesthesia has been suggested as a risk factor for this frightening phenomenon [28].

As is the case with perception of pain, a patient's awareness is perceived differently by caregivers and family members. In a study on patients in a persistent vegetative state, $90 \%$ of these patients were considered by family members to have some awareness of the family member's presence and of pain [29]. The study on patients with locked-in syndrome also showed that the first person to realize the patient was conscious and could communicate with eye movements was most often a family member (55\% of cases) and not the treating physician ( $23 \%$ of cases) [27].

The above examples show how difficult it is to recognize unambiguous signs of conscious perception of the environment and the self. This also applies to the assessment of awareness and communication in non-verbally expressive end-stage palliative sedation patients, who traditionally do not benefit from detailed neurological examinations.

In contrast to palliative care settings, in which advanced monitoring equipment is avoided, in the ICU bispectral (BIS) monitors are usually available. The BIS monitor is a reliable instrument for assessment of the level of sedation. Low BIS index values are known to correlate negatively with higher sedative concentrations. It has to be remembered that assessment of anesthetic depth is not the same as assessment of comfort [30]. Some studies suggest that use of BIS monitoring may be useful, es- 
pecially in patients who have been administered blocking agents [9]. As mentioned above, behavioral observation is unreliable in these patients because even patients in severe pain may show no observable signs of distress. In a study in non-verbal ICU patients who were mechanically ventilated and sedated, the BIS index seemed to be an indicator for pain during procedures known to be painful. However, further research is needed before this technique can be used for the detection of pain in non-communicative patients [9].

Based on these findings, we have to realize that 'traditional' clinical tools and procedures to assess comfort and awareness in dying non-communicative patients have important methodological limitations. It should be noted that the problems with assessments are not to be ascribed to lack of competence on the part of the caregivers but are of a much more fundamental nature: The absence of reliable tools. The developers of guidelines for palliative sedation are aware of these limitations and rightly point out that there is a lack of evidence (Box 1). Some guidelines mention that "there are no scales available to assess the patient's comfort" [31] and the authors of a recent guideline conclude that "presently no particular scale can be recommended" [16]. Sometimes guidelines refer to sedation scales but point out that these scales are "not intended to measure the effect of sedation but to make clear when the sedation is too deep" [31]. The current guidelines for palliative sedation are therefore limited to suggesting "a daily visit by the physician" and "continue attention to possible expressions of discomfort (e.g., facial expressions, movements etc.)" [31, 32]. Not surprisingly, people who have more intensive contact with the patient, such as nurses and family members, should also play an important role in signaling discomfort in sedated patients [31].

\section{Problems with the Titration of Drugs}

Since the aim of palliative sedation is to give optimal comfort but not to hasten death, the principle of proportionality is a pivotal aspect of this treatment. Hence, the guidelines state that sedation should be "no deeper than necessary to avoid suffering" $[15,16,31,32]$. To meet this principle of proportionality, caregivers should carefully titrate the doses of the drugs so that they are high enough to provide comfort but should not hasten death. Studies have shown that palliative sedation does not usually affect survival time [5]. However, the fact that palliative sedation is considered by some to be 'slow euthanasia' may lead physicians to be 'extra careful' with the use of high doses of sedative medication [4]. Several studies have reported underuse of medicines because of a lack of knowledge, unwarranted beliefs, to avoid the perception of giving 'excessive' doses and even because of fear among caregivers of being accused of 'killing' the patient [33, 34]. In a Dutch study among nurses, sedation was considered insufficiently effective by $42 \%$ of the respondents [35]. 


\section{Recent Advances Changing Our View of Palliative Sedation}

In recent years important progress has been made that has forced us to review our opinions about comfort during palliative sedation. We will report here on some major breakthroughs in the assessment of pain, the assessment of consciousness and the mechanisms of sedation.

An important insight with regard to pain perception and brain activity is that several areas in the brain (thalamus, S1, and the secondary somatosensory or insular, frontoparietal, and anterior cingulate cortices) are activated by pain. This neurological signature of pain is also known as the pain matrix [36]. In a study with patients in a minimally conscious state, patients in a persistent vegetative state and healthy volunteers, Boly and colleagues investigated brain activation induced by noxious stimulation [37]. A remarkable finding was that in minimally conscious state patients, no area of the pain matrix was less activated than in the healthy control group. Since cerebral correlates of pain processing are found in a similar network in controls and patients with minimally conscious state, it is plausible that patients in a minimally conscious state experience pain. An important benefit of this method is that it provides objective evidence of potential pain perception and does not require communication with the patient. This method is also promising because cerebral activity is not only a good indicator for physical pain but can also be considered as an objective indicator for other kinds of suffering, such as emotional suffering caused by feelings of rejection [36]. This may be important because in palliative care all the dimensions of suffering, i.e., physical, social, emotional and spiritual suffering, must be evaluated.

A second breakthrough to be mentioned occurred in the field of awareness. Despite its widespread use, little is known about how anesthesia produces loss of consciousness. Over the past five years, significant progress has been made in understanding what happens in the brain as consciousness departs and returns [38]. Studies based on neuroimaging and electroencephalography have found evidence to support the integrated information theory. This theory states that consciousness depends on the brain's capacity to sustain complex patterns of internal communication $[39,40]$. Hence, if we are able to measure communication between different brain areas, this may be an indication of the degree of consciousness. A possible method of establishing communication between brain areas is based on the use of transcranial magnetic stimulation (TMS) and high density EEG. Based on this idea, a recent study introduced a theory-driven index of the level of consciousness, called the perturbational complexity index. This index was tested on a large data set of TMS-evoked potentials recorded with high density EEG in healthy patients and patients with consciousness disorders. The index that resulted from this study can potentially be used for objective determination of the level of consciousness at the bedside [41].

In a study by Boly et al. with healthy volunteers, high density EEG recordings were made during gradual induction of and emerging from unconsciousness with propofol. The study revealed structured EEG patterns that mark the transition between consciousness and unconsciousness induced by propofol. These findings 
provide precise, neurophysiologically principled markers that can be used to monitor the state of a patient's unconsciousness under propofol general anesthesia [42].

Given the important ethical and clinical aspects related to the depth of sedation, adequate sedation is considered a crucial aspect of palliative sedation and all the guidelines highlight its importance (Box 1). Since the guidelines all seems to rely on behavioral observation, they are based on an important premise, i. e., that the unresponsiveness of the patient is proof of unawareness, and hence of the absence of distress. However, it appears that this premise should be questioned. In an important publication, Sanders et al. concluded that unresponsiveness was not the same as unconsciousness [13]. Unresponsiveness sometimes provides unreliable information about the probability of consciousness. To achieve optimal titration during palliative sedation, i. e., to ensure that the patient is comfortable and to avoid unnecessarily high levels of sedation, we need to make a distinction between three separate concepts: Consciousness, connectedness and responsiveness.

Consciousness can be connected or disconnected. In the latter case, a person can be conscious without experiencing the external world. An example of this is dreaming during natural sleep, but dreaming also seems to occur in more than a quarter of patients anesthetized with propofol or desflurane [43]. Another finding reported by Sanders et al. is based on the isolated forearm technique (IFT). In the IFT, anesthesia is followed by the inflation of a cuff on the arm before neuromuscular blockade is induced. The cuff prevents paralysis of the hand and hence allows a simple kind of communication through predefined hand movements, for instance, squeezing the hand if the answer to a question is "yes". A literature review of IFT studies found that positive responses were observed in a median of $37 \%$ of patients, which is evidence of connected consciousness [13]. Strikingly, also, EEG monitors based on the depth of anesthesia, such as the BIS monitor, could not reliably distinguish between responders and non-responders to the IFT [44, 45].

The above findings have practical implications for anesthesia and palliative sedation, e.g., for monitoring the depth of sedation and the titration of drugs used for palliative sedation. One implication is that consciousness should not always be a clinical problem in which the subjective experience of pain and other kinds of distress is disconnected. If we do not make the distinction between the three concepts responsiveness, consciousness and connectedness - we risk either administering doses of sedatives that may be too low, entailing the risk of an uncomfortable death, or too high, entailing the risk that this act is perceived as life-shortening or even euthanasia. An example of a case in which insufficient sedation may occur is in patients who are unresponsive but may nevertheless retain some kind of consciousness and hence are able to feel pain and distress. An example in which patients do not necessarily need higher levels of sedation, notwithstanding the fact that they do have some kind of consciousness, may occur when their consciousness is disconnected, which implies that they do not experience their suffering. In theory, this may happen when (palliative) sedated patients are dreaming. Such an event can be detected with an EEG showing the typical pattern of rapid eye movement (REM) sleep, which is similar to the EEG pattern when people are awake. However, we do not have sufficient understanding of how and when consciousness becomes con- 
nected to the environment, especially in dying, palliative sedated patients who are very difficult to include in studies on this topic. Moreover, patients on palliative sedation eventually die and will by definition not be able to remember their experience.

Another problem is that palliative sedated patients have not been involved in any of the above studies. This is understandable, because it is ethically delicate to include such patients in these studies. However, given the possible impact, efforts should be made to design studies that do not impose too much extra burden on these patients so that they can be included. Even studies with a limited number of selected patients would be very useful.

\section{How Can the Assessment of Suffering in Palliative Sedated Patients be Improved?}

Until now, studies of the efficacy of palliative sedation to relieve pain and discomfort have been based on observational scales or subjective assessments by caregivers [35]. Although some efforts have been made to validate the observation scales, as far as we know all these attempts are based on the same paradigm, i.e., that all kinds of distress in all patients can be measured by observation of the patient and that this is the only available method.

As illustrated in the above studies, new insight and technologies, such as fMRI and EEG, have proven to be promising technologies for detecting awareness and pain that cannot be observed or detected by 'traditional' methods [25]. Although these technologies also have their limitations and should not be regarded as a perfect surrogate for self-reporting, they provide valuable objective and quantifiable indicators of awareness and pain in non-communicative patients. Strikingly, they have not yet been used to check whether the current assessments of palliative sedated patients are reliable. It is remarkable that, given the increasing incidence of palliative sedation, there is so little concern about the risks that patients may experience an uncomfortable dying phase in which they are unable to signal their suffering. An assessment tool that would allow clinicians to determine the appropriate doses of medications more accurately would also encourage more vigorous symptom management in the dying.

Paradoxically, the inability to report distress might also be aggravated or even blocked by the use of drugs that may abolish potential further communication and even facial expressions [12]. Hence, some patients might have subjective phenomenological awareness or suffering with very limited, fluctuating or absent behavioral motor signs of distress [13]. The fact that neuroimaging or electrophysiology recordings have not been used so far to validate the assessment tools for distress in non-communicative patients, even when doubts about these tools have arisen, may be related to the reluctance in palliative and end-of-life care to bother patients with high-tech equipment as, in most cases, patients have already experienced a long treatment period. 


\section{Conclusions}

A problem in monitoring the depth of palliative sedation and the titration of sedatives is that caregivers still have to make use of very rudimentary assessment tools, which do not take into account the above described new insights. Uncommunicative dying patients are a vulnerable population and, therefore, we should do everything possible to assure them a comfortable death, free of pain and distress. We, therefore, urgently need a triangulation of methods in which existing observational scales, subjective assessments of caregivers and family, and neuroimaging and/or electrophysiological techniques are combined (Fig. 1). The latter are non-invasive procedures which should not burden the patient and his/her family too much. Because of its complexity and intensity, this integrated mixed method is intended for research and not (yet) for everyday clinical assessments. It can be used for the validation of existing clinical tools for the assessment of distress in palliative sedated patients. Each of the three methods has its potentials and limitations but, in combination, they can be used to achieve the best possible assessments.

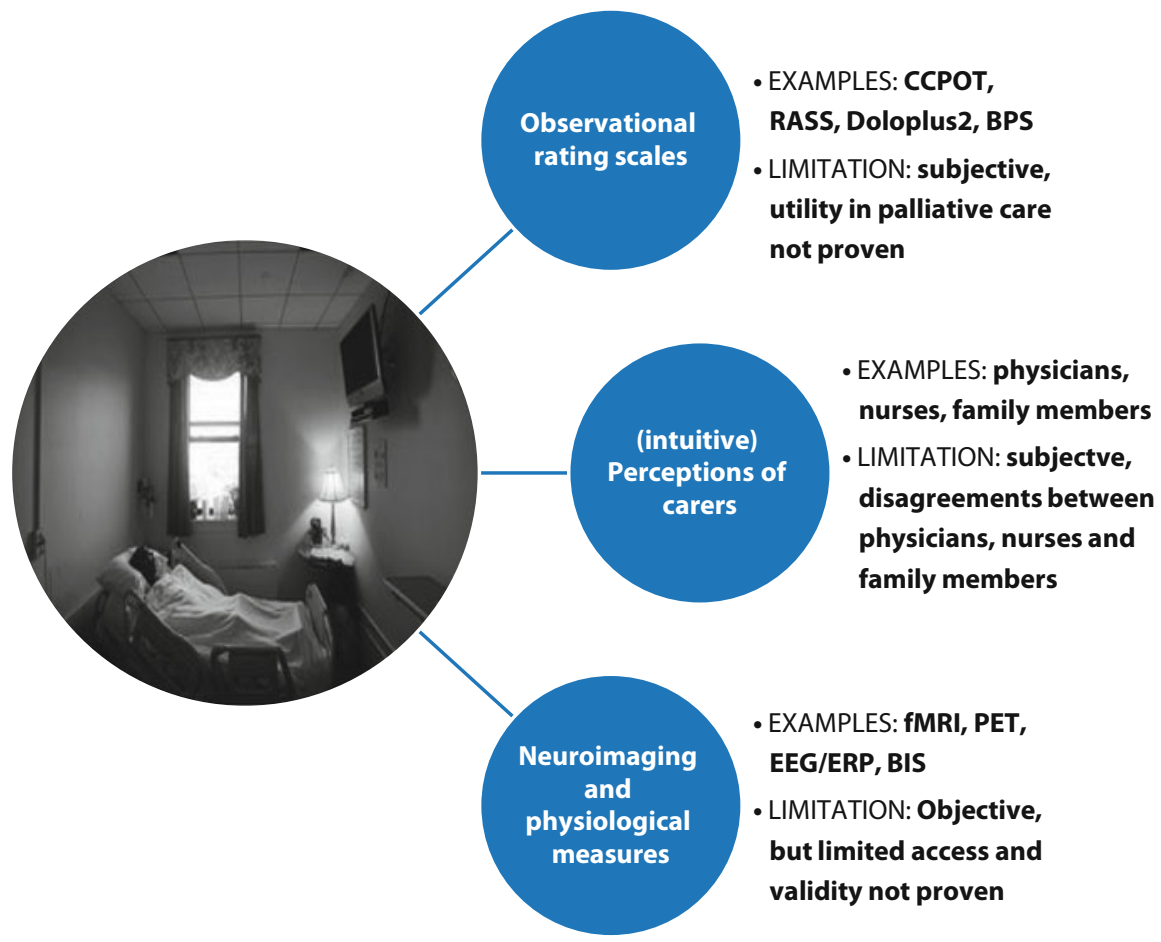

Fig. 1 Triangulation of assessment of distress in the non-communicative dying patient (adapted from [2] with permission). CCPOT: Critical Care Pain Observational Tool; RASS: Richmond Agitation-Sedation Scale; BPS: Behavioral Pain Scale; fMRI: functional magnetic resonance imaging; PET: positron emission tomography; ERP: event related potential; EEG: electroencephalography; BIS: bispectral index 


\section{Acknowledgements}

Supported by a grant from the Research Council of the Vrije Universiteit Brussel (Project HOA 27). SL is Research Director at the Belgian National Fund for Scientific Research (FRS). The authors also want to thank Said Hachimi-Idrissi, Jan Poelaert and Wim Distelmans for their valuable input.

\section{References}

1. Gelinas C (2007) Management of pain in cardiac surgery ICU patients: have we improved over time? Intensive Crit Care Nurs 23:298-303

2. Deschepper R, Laureys S, Hachimi-Idrissi S, Poelaert J, Distelmans W, Bilsen J (2013) Palliative sedation: Why we should be more concerned about the risks that patients experience an uncomfortable death. Pain 154:1505-1508

3. Kirk TW, Mahon MM (2010) National Hospice and Palliative Care Organization (NHPCO) position statement and commentary on the use of palliative sedation in imminently dying terminally ill patients. J Pain Symptom Manage 39:914-923

4. Claessens P, Menten J, Schotsmans P, Broeckaert B (2012) Level of consciousness in dying patients. The role of palliative sedation: a longitudinal prospective study. Am J Hosp Palliat Care 29:195-200

5. Maltoni M, Scarpi E, Rosati M et al (2012) Palliative sedation in end-of-life care and survival: a systematic review. J Clin Oncol 30:3429

6. Rys S, Mortier F, Deliens L, Deschepper R, Battin MP, Bilsen J (2013) Continuous sedation until death: moral justifications of physicians and nurses - a content analysis of opinion pieces. Med Health Care Philos 16:533-542

7. Anquinet L, Rietjens JA, Seale C, Seymour J, Deliens L, van der Heide A (2012) The practice of continuous deep sedation until death in Flanders (Belgium), the Netherlands, and the U.K.: a comparative study. J Pain Symptom Manage 44:33-43

8. Demertzi A, Racine E, Bruno MA et al (2013) Pain perception in disorders of consciousness: neuroscience, clinical care, and ethics in dialogue. Neuroethics 6:37-50

9. Gelinas C, Tousignant-Laflamme Y, Tanguay A, Bourgault P (2011) Exploring the validity of the bispectral index, the Critical-Care Pain Observation Tool and vital signs for the detection of pain in sedated and mechanically ventilated critically ill adults: a pilot study. Intensive Crit Care Nurs 27:46-52

10. Ahlers SJ, van der Veen AM, van Dijk M, Tibboel D, Knibbe CA (2010) The use of the Behavioral Pain Scale to assess pain in conscious sedated patients. Anesth Analg 110:127133

11. Arevalo JJ, Brinkkemper T, van der Heide A et al (2012) Palliative sedation: reliability and validity of sedation scales. J Pain Symptom Manage 44:704-714

12. Brown JE, Chatterjee N, Younger J, Mackey S (2011) Towards a physiology-based measure of pain: patterns of human brain activity distinguish painful from non-painful thermal stimulation. PLoS One 6:e24124

13. Sanders RD, Tononi G, Laureys S, Sleigh JW (2012) Unresponsiveness not equal unconsciousness. Anesthesiology 116:946-959

14. Brinkkemper T, van Norel AM, Szadek KM, Loer SA, Zuurmond WW, Perez RS (2013) The use of observational scales to monitor symptom control and depth of sedation in patients requiring palliative sedation: a systematic review. Palliat Med 27:54-67

15. de Graeff A, Dean M (2007) Palliative sedation therapy in the last weeks of life: a literature review and recommendations for standards. J Palliat Med 10:67-85

16. Dean MM, Cellarius V, Henry B, Oneschuk D (2012) Framework for continuous palliative sedation therapy in Canada. J Palliat Med 15:870-879 
17. Kappesser J, Williams AC (2010) Pain estimation: asking the right questions. Pain 148:184187

18. Breau LM, McGrath PJ, Stevens B et al (2006) Judgments of pain in the neonatal intensive care setting: a survey of direct care staffs' perceptions of pain in infants at risk for neurological impairment. Clin J Pain 22:122-129

19. Demertzi A, Schnakers C, Ledoux D et al (2009) Different beliefs about pain perception in the vegetative and minimally conscious states: a European survey of medical and paramedical professionals. Prog Brain Res 177:329-338

20. Platts-Mills TF, Hunold KM, Weaver MA et al (2013) Pain treatment for older adults during prehospital emergency care: variations by patient gender and pain severity. J Pain 14:966-974

21. Halliburton JR (1998) Awareness during general anesthesia: new technology for an old problem. CRNA 9:39-43

22. Laureys S (2005) Science and society: death, unconsciousness and the brain. Nature Rev Neurosci 6:899-909

23. Schnakers C, Vanhaudenhuyse A, Giacino J et al (2009) Diagnostic accuracy of the vegetative and minimally conscious state: clinical consensus versus standardized neurobehavioral assessment. BMC Neurol 9:35

24. Cruse D, Chennu S, Chatelle C et al (2011) Bedside detection of awareness in the vegetative state: a cohort study. Lancet 378:2088-2094

25. Monti MM, Vanhaudenhuyse A, Coleman et al (2010) Willful modulation of brain activity in disorders of consciousness. N Engl J Med 362:579-589

26. Laureys S, Schiff ND (2012) Coma and consciousness: paradigms (re)framed by neuroimaging. Neuroimage 61:478-491

27. Laureys S, Pellas F, Van Eeckhout $P$ et al (2005) The locked-in syndrome: what is it like to be conscious but paralyzed and voiceless? Prog Brain Res 150:495-511

28. Ghoneim MM, Block RI, Haffarnan M, Mathews MJ (2009) Awareness during anesthesia: risk factors, causes and sequelae: a review of reported cases in the literature. Anesth Analg 108:527-535

29. Tresch DD, Sims FH, Duthie EH Jr, Goldstein MD (1991) Patients in a persistent vegetative state attitudes and reactions of family members. J Am Geriatr Soc 39:17-21

30. Sackey PV (2008) Frontal EEG for intensive care unit sedation: treating numbers or patients? Crit Care 12:186

31. Royal Dutch Medical Association (KNMG) (2009) Guideline for palliative sedation. Available at: http://www.palliativedrugs.com/download/090916_KNMG_Guideline_for_ Palliative_sedation_2009_2_\%5B1\%5D.pdf. Accessed Nov 2013

32. Cherny NI, Radbruch L (2009) European Association for Palliative Care (EAPC) recommended framework for the use of sedation in palliative care. Palliat Med 23:581-593

33. Jackson DL, Proudfoot CW, Cann KF, Walsh TS (2009) The incidence of sub-optimal sedation in the ICU: a systematic review. Crit Care 13:R204

34. Oldenmenger WH, Sillevis Smitt PA, van Dooren S, Stoter G, van der Rijt CC (2009) A systematic review on barriers hindering adequate cancer pain management and interventions to reduce them: a critical appraisal. Eur J Cancer 45:1370-1380

35. Brinkkemper T, Klinkenberg M, Deliens L et al (2011) Palliative sedation at home in the Netherlands: a nationwide survey among nurses. J Adv Nurs 67:1719-1728

36. Wager TD, Atlas LY, Lindquist MA, Roy M, Woo CW, Kross E (2013) An fMRI-based neurologic signature of physical pain. N Engl J Med 368:1388-1397

37. Boly M, Faymonville ME, Schnakers C et al (2008) Perception of pain in the minimally conscious state with PET activation: an observational study. Lancet Neurol 7:1013-1020

38. Bodart O, Laureys S, Gosseries O (2013) Coma and disorders of consciousness: scientific advances and practical considerations for clinicians. Semin Neurol 33:83-90

39. Rosanova M, Gosseries O, Casarotto S et al (2012) Recovery of cortical effective connectivity and recovery of consciousness in vegetative patients. Brain 135:1308-1320 
40. Demertzi A, Soddu A, Laureys S (2013) Consciousness supporting networks. Curr Opin Neurobiol 23:239-244

41. Casali AG, Gosseries O, Rosanova M et al (2013) A theoretically based index of consciousness independent of sensory processing and behavior. Sci Transl Med 14:198ra105

42. Boly M, Moran R, Murphy M et al (2012) Connectivity changes underlying spectral EEG changes during propofol-induced loss of consciousness. J Neurosci 16:7082-7090

43. Leslie K, Sleigh J, Paech M, Voss M, Lim C, Sleigh C (2009) Dreaming and electrographic changes during anesthesia maintained with propofol or desflurane. Anesthesiology 111:547555

44. Schneider G, Wagner K, Reeker W et al (2002) Bispectral Index (BIS) may not predict awareness reaction to intubation in surgical patients. J Neurosurg Anesthesiol 14:7-11

45. Russell IF (2006) The Narcotrend 'depth of anaesthesia' monitor cannot reliably detect consciousness during general anaesthesia: an investigation using the isolated forearm technique. Br J Anaesth 96:346-352 